Revue d'histoire du XIXe siècle

Société d'histoire de la révolution de 1848 et des

révolutions du XIXe siècle

$28 \mid 2004$

Religion, politique et culture au XIXe siècle

\title{
Être insurgé et être citoyen à Paris durant la Seconde République
}

Thèse de doctorat en histoire sous la direction d'Alain Corbin, Université de Paris 1-Panthéon-Sorbonne, 947 fo., soutenue le 14 novembre 2003 devant un jury composé de Philippe Boutry, Claude-Isabelle Brelot (présidente), Alain Corbin, Jean-Claude Caron, Dominique Kalifa.

\section{Louis Hincker}

\section{OpenEdition}

\section{Journals}

Édition électronique

URL : http://journals.openedition.org/rh19/682

DOI : $10.4000 /$ rh 19.682

ISSN : $1777-5329$

Éditeur

La Société de 1848

Édition imprimée

Date de publication : 1 juin 2004

Pagination : 223-229

ISSN : 1265-1354

Référence électronique

Louis Hincker, "Être insurgé et être citoyen à Paris durant la Seconde République », Revue d'histoire du XIXe siècle [En ligne], 28 | 2004, mis en ligne le 20 juin 2005, consulté le 10 décembre 2020. URL: http://journals.openedition.org/rh19/682 ; DOI : https://doi.org/10.4000/rh19.682

Ce document a été généré automatiquement le 10 décembre 2020.

Tous droits réservés 


\section{Être insurgé et être citoyen à Paris durant la Seconde République}

Thèse de doctorat en histoire sous la direction d'Alain Corbin, Université de Paris 1-Panthéon-Sorbonne, $947 \mathrm{f}^{\circ}$., soutenue le 14 novembre 2003 devant un jury composé de Philippe Boutry, Claude-Isabelle Brelot (présidente), Alain Corbin, Jean-Claude Caron, Dominique Kalifa.

\section{Louis Hincker}

1 Cette recherche est née d'une opportunité documentaire : au lendemain de chacune des journées révolutionnaires parisiennes de la Seconde République, les différentes autorités qui se succèdent mettent en place des procédures de récompense, de répression, et d'indemnisation. Ces procédures se présentent sous la forme de séries de dossiers individuels, de dossiers nominatifs : récompense et indemnisation pour les combattants et blessés de la révolution de février 1848 d'abord, répression pour le 15 mai 1848 ensuite - jour d'envahissement de l'Assemblée nationale qui vient d'être élue -, répression encore pour les journées de juin 1848 - nouvelles journées barricadières quatre mois après la révolution -, répression de nouveau pour le 13 juin 1849 - manifestation du « parti » démocrate-socialiste qui tourne à l'affrontement -, répression enfin pour les 3 et 4 décembre 1851 - jours d'une résistance non négligeable à Paris au coup d'État de Louis-Napoléon Bonaparte. Il faut ajouter l'indemnisation, trente ans après les faits, des «victimes du 2 décembre 1851 » par la loi du 30 juillet 1881 qui s'adresse aux survivants mais aussi à leurs veuves ou à leurs descendants : loi à l'initiative de la Troisième République qui répare les dommages mais n'accorde pas des récompenses. Si l'on ajoute les différentes demandes de grâce, on parvient à un ensemble d'environ 20000 dossiers individuels se rapportant aux participants des journées révolutionnaires de l'époque.

2 La figure de l'« insurgé », aussi bien en 1848 que sous la plume des historiens, a occulté l'expérience de celui que nous avons choisi de nommer par l'expression de «citoyencombattant ». Pour le comprendre, il n'y pas d'autre solution que de rouvrir les dossiers d'archives des participants aux barricades. Notre travail se présente non pas comme la réhabilitation de ce statut qui n'a pas d'équivalent dans notre démocratie actuelle mais 
comme sa restitution pour une meilleure compréhension des journées révolutionnaires. À l'époque, le sentiment de légalisme apparaît comme bien plus profond, durable, et répandu, que le désir de subversion, en particulier les armes à la main au cœur des combats, grâce à la nouvelle garde nationale née de la révolution de février 1848.

3 Pour autant le citoyen-combattant, un temps honoré, connaît une répression irrémédiable et perd sa légitimité dès l'été 1848. «Citoyens » et «insurgés » donc, en même temps ou successivement, ce sera selon le jugement porté par les uns ou par les autres, tel sont les qualificatifs de ceux qui ont retenu notre attention. Leur manière de s'accommoder de sentences opposées et contradictoires est le principal objet de notre réflexion. Surtout, le devenir sur le plus ou moins long terme, toujours sous la contrainte, permet de voir jusqu'à quel point l'expérience des combats perdurent une fois les événements terminés. La valeur, recomposée à différentes étapes de l'existence, que chacun accorde à ses faits et gestes dit encore la participation de jadis, et parfois jusque dans les vieux jours.

4 À travers les procédures que nous avons étudiées, la « journée insurrectionnelle » est autant valorisée que stigmatisée. Ces procédures cherchent à saluer le mérite, à rendre hommage, à reconnaître l'honneur, à secourir le survivant, à indemniser la victime, mais aussi à traquer ou gracier le responsable à la suite d'une répression. Des comparaisons s'imposent pour comprendre leurs finalités différentes, car il y a loin de la récompense honorifique au secours pécuniaire, de la pension à la peine de prison. Mais il importe de souligner ce qu'elles en commun, quand elles s'adressent à chaque « insurgé » en particulier au nom d'un ordre politique auquel elles se réfèrent, quand elles manipulent les catégories du droit pénal. Elles évaluent des qualités, elles prennent en charge des destinées, elles délimitent des frontières entre les sphères du public et du privé, elles définissent les normes de la citoyenneté. Chacune participe d'une politique de la mémoire et de l'oubli, instrumentalisés et prescrits, et par conséquent d'une d'épuration du souvenir, car elles cherchent à rétablir l'union entre les citoyens et à rejeter dans le passé la figure de «l'insurgé ». À chaque fois, les mêmes procédés sont utilisés par l'administration : des listes de noms, des fichiers, et surtout des dossiers individuels.

5 La nature comme l'ampleur de la documentation nous ont amené à construire de manière raisonnée notre enquête (1 ${ }^{\text {ère }}$ partie). D'une part, il s'agissait de ne pas concevoir l'écriture des dossiers comme un instrument transparent de médiation entre les mots et les choses. Le requérant ne pouvait être appréhendé que dans sa manière d'utiliser la fonction de représentation de l'écriture. D'autre part, nous n'avons jamais envisagé une analyse exhaustive des dossiers, sans grande signification et de toute façon irréalisable. 159 personnes, pour lesquelles nous avons réuni 385 dossiers, ont finalement été retenues. Nous avons choisi de ne sélectionner que les individus ayant un dossier dans plusieurs de ces procédures. Le travail a consisté à étudier particulièrement ceux ayant, d'une part, participé de la légitimation du combattant des journées révolutionnaires et ayant cherché à en bénéficier par la récompense ou l'indemnisation, mais ayant d'autre part tout aussi bien connu la dénégation de cette légitimité lors de l'inculpation. Ce corpus, volontairement restreint, est le fruit de critères de sélections, il n'est en rien aléatoire. Il permet d'envisager l'articulation entre appartenances, expériences et aspirations, des citoyens-combattants, autrement dit leur socialisation politique à la lumière des journées révolutionnaires. 
6 C'est à l'identité institutionnelle du requérant, jamais analysée en tant que telle par l'historiographie des événements, que nous nous sommes ensuite consacré ( $2{ }^{\text {ème }}$ partie). La relation contrainte avec les autorités engage durablement les devenirs. Mais l'autorité du droit n'est pas que défavorable au citoyen-combattant même dans le cadre de la répression des journées révolutionnaires. Son statut de sujet de droit peut le protéger, ainsi qu'une partie de ses «proches». Les lendemains des événements révèlent l'importance de la famille, à la fois ressource et refuge pour celui qui dit s'être autant placé au service des siens que de la cité. Les dossiers éclairent ce lien familial, son pouvoir légitimant face à l'adversité, et dessinent la relation étroite, mal perçue jusqu'ici par les historiens, entre le statut de père et celui du citoyen-combattant, entre le protecteur du foyer et le défenseur de la République.

7 Les dossiers ne se réduisent pas à une confrontation codifiée et stéréotypée. Leur contenu reste en relation avec les événements. Les justifications et les revendications détournent les procédures de leurs fonctions premières. La légitimité des faits et gestes est remise en jeu, le face-à-face entre requérants et autorités procède par un recouvrement de l'ordre socio-politique par l'ordre juridique qui n'est pas sans faille. Les mots, instables, aux significations sans cesse renégociées, font affleurer l'expérience des combats. Nous avons donc tenté de nous rapprocher ainsi d'un peu plus près des barricades et des foyers ( $3^{\text {ème }}$ partie), d'en comprendre surtout les différentes logiques en profitant de la grande variété sociologique de notre corpus de citoyens-combattants.

8 Les armes citoyennes en 1848 sont dans les mains des hommes. Le caractère sexué de la garde nationale démocratisée traduit un ensemble de règles sociales et souligne une inégale familiarité avec le fusil, avec l'habitude du commandement, ainsi que l'exclusion des femmes de cet exercice particulier de la souveraineté. La mobilisation citoyenne parfois s'improvise, mais les institutions qui entendent l'encadrer sont le produit d'une "culture des armes » qui définit pour chacun, officiers de la garde nationale comme simples combattants, maris comme épouses, leur droits et leurs devoirs. Cependant l'expérience des événements offre une large place aux initiatives personnelles. C'est précisément la liberté que s'autorise l'homme en armes qui perd dès l'été 1848 sa légitimité.

9 La révolution de 1848 offre à tous de nouvelles expériences politiques en même temps

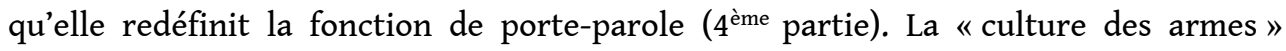
favorise les officiers de la garde nationale. Mais ceux-ci entrent parfois en concurrence avec leurs contemporains : les délégués ouvriers et les hommes politiques. L'apprentissage de l'illégitimité politique de la prise d'armes sur le long terme, qui vise d'abord à neutraliser la «culture des armes » des citoyens-combattants, atteint différemment les porte-parole de la révolution. Disparaissent ceux qui sont trop dépendants des circonstances des journées révolutionnaires et qui ne correspondent pas à la réaffirmation de la nécessaire capacité politique qui fait le citoyen.

10 Notre démarche n'a pas comme horizon de proposer une typologie des citoyenscombattants. Elle souligne simplement les écarts croissants au fil des événements entre les différents impétrants. Elle propose, grâce au portrait singulier, de lire les logiques de la participation politique jusque dans leurs aspects les plus contradictoires (5 ${ }^{\text {ème }}$ partie). L'avènement éphémère d'une forme de citoyenneté gagne ainsi à être perçue au regard des manières dont chacun entend en être et en rester partie prenante, même une fois perdue sa légitimité. 
11 Qu'est-ce alors que le « citoyen-combattant ", expression qui est avant tout la nôtre? Nous dirons : un « devoir être » depuis les journées de février 1848, vis-à-vis duquel à l'époque chacun se situe et ordonne ses expériences des événements. Puis, le modèle laisse place ensuite à l'obligation de se déprendre de toute revendication à un quelconque droit au fusil. Le « citoyen-combattant ", c'est aussi le contre-modèle qu'il est devenu. C'est bien pourquoi il ne faut pas le confondre avec le « soldat-citoyen » qui s'impose en profitant de la répression des journées révolutionnaires parce que bras armé du pouvoir exécutif par opposition à celui de la « société civile » incarné par la garde nationale démocratisée. Sur ce point la Seconde République est un tournant capital. Les démocrates-socialistes, échaudés par l'année 1848, délaissent le citoyencombattant pour capter le suffrage des militaires. Seul l'éthique de la «défense nationale » peut expliquer la résurgence de la prise d'armes citoyenne de septembre 1870 à mai 1871, mais soulignons l'absence de celle-ci dans l'affirmation du droit au fusil en février comme en juin 1848 ou encore en décembre 1851 ; autrement dit : pas de «levée en masse» sous la Seconde République, mais un exercice particulier de la souveraineté fusil en main.

12 Une procédure comme celle qui indemnise les «victimes du 2 décembre » durant les premières années de la Troisième République tend à unifier le temps de la citoyenneté pour parer aux effets centrifuges des temporalités singulières de chacun des trajets personnels. À plus long terme, la temporalité propre aux journées révolutionnaires disparaît, et la trace qu'en porte chacun des participants s'estompe. Quelques années de plus, et le décès symbolise la disparition d'une forme de citoyenneté révolue. Comme au temps des années 1848-1851, ce type de politique du temps définit des modes de socialisation en articulant les appartenances et les aspirations. C'est un réel pouvoir que de déclarer quand tel ou tel peut enfin devenir citoyen. Cette conception d'un apprentissage nécessitant une certaine durée préalable d'incubation s'oppose à l'immédiateté d'une journée révolutionnaire qui offre à tous la possibilité de participer à l'espace public ici et maintenant. L'indemnisation des "victimes du 2 décembre » ne réhabilite pas la révolution de 1848. Plus généralement, toutes les procédures que nous avons analysées montrent comment l'apprentissage de la démocratie s'accompagne de l'apprentissage corollaire de l'illégitimité de la prise d'armes citoyenne.

13 Les dossiers des combattants parlent surtout des lendemains des combats. Mais il est selon nous possible de retrouver la signification perdue de la participation aux barricades. Il faut par exemple commencer par distinguer la « mémoire historique » de la révolution de 1848 ou de la résistance au coup d'État, telle qu'elle est définie, prescrite et instrumentalisées par le débat parlementaire des années 1880, qui rejette dans l'illégitimité la figure de l'«insurgé», qui ne parle que de "blessés» ou de « victimes », qui ne voit que des martyrs défendant le droit. Il y a ensuite la "mémoire vive » des anciens citoyens-combattants, obligés le plus souvent de taire les aspects les plus subversifs de leurs expériences des combats. Il y a enfin la mémoire recomposée par les veuves et les descendants qui vivent encore le lourd tribut de la participation aux barricades et pour qui le déclassement est le mode vécu de l'apprentissage de l'illégitimité politique de la prise d'armes citoyenne. Eux ne répètent ni ne reproduisent les expériences ; c'est à peine si les significations se transmettent d'une génération à l'autre. Il y a écarts et différences donc, mais pas de stricte séparation entre tous ceux qui portent ou prennent en charge le devenir des expériences révolutionnaires de la Seconde République. Il faut donc considérer la spécificité de la première génération des 
descendants des quarante-huitards pour qui l'héritage est encore en souffrance, dans les deux sens de cette expression : douloureux et dans l'attente d'une réparation.

La famille qui transparaît dans les dossiers individuels, c'est d'abord celle convoquée par les autorités ; elle ne porte pas les armes ; elle est associée soit à la reconnaissance du sacrifice soit à l'illégitimité du citoyen-combattant. Mais puisqu'elle est sollicitée, elle fait valoir qu'elle est à la base de la « société civile » - c'est ce qu'on lui demande. Elle montre la relative autonomie de son pouvoir légitimant - c'est ce qu'on est bien obligé de lui reconnaître même de façon mesurée. Par quelles voies la famille est-elle donc productrice de citoyenneté? Si on a l'habitude d'appréhender les rapports famille/Cité - et notamment le contrôle de la seconde sur la première - à partir des discours juridiques, politiques, philosophiques, pédagogiques, littéraires, et religieux, notre travail montre l'intérêt qu'il y a à procéder en essayant d'approcher la texture du lien familial entre des personnes participant d'une même économie domestique. Nous avons bien conscience de n'avoir qu'essayé, mais nous pensons qu'il y a là bien des possibilités de comprendre l'investissement civique et politique notamment des milieux populaires. Le citoyen-combattant père de famille, par exemple, n'a pas pour charge de représenter ni d'incarner ses "proches» les armes à la main. Il est simplement en relation avec eux, il les protège, mais la limite de son engagement au service de la cité est atteinte quand il risque de mettre en danger les siens. Le combattant s'identifie au citoyen-sauveteur, au service de ses " proches » et du bien public. Son patriotisme reste relié au statut du chef de famille. Et s'il faut bien souligner que les épouses ne sont pas concernées par le port de l'arme, il faut aussi noter qu'elles ne le vivent pas comme une exclusion; nous n'avons pas rencontré de trace d'une quelconque revendication du droit au fusil de leur part.

La répression des journées révolutionnaires de la Seconde République n'abolit pas la citoyenneté, elle la refonde. Les " prévenus d'insurrection » ainsi que leurs «proches » sont même conviés à cette œuvre de régénération. Répression de classe, assurément, mais selon quelles visées? À trop se focaliser sur les Ateliers nationaux en juin 1848, l'historiographie est passée à côté de l'essentiel. Ce n'est pas tant les ouvriers qui sont alors réprimés mais une forme de citoyenneté qui a les faveurs des milieux populaires parisiens. Leur manière d'être dans l'espace civique est qualifiée de « délit politique ». Car la prise d'armes favorise toutes sortes d'expériences politiques en période révolutionnaire. La participation personnelle, mêlant étroitement sentiments, conventions et opinions, valorisant le dévouement, permettant de se forger son propre jugement et de se considérer comme citoyen à part entière, en dépit des difficultés socio-économiques rencontrées, encadrée par la garde nationale démocratisée, doit finalement laisser place à la refondation de la citoyenneté capacitaire. Et ceux, hommes politiques et hommes de la classe moyenne, qui sont accusés d'avoir dérogé à leur position sociale connaissent comme les autres la prison, la transportation et l'exil dès les lendemains de juin 1848 et plus encore après le coup d'État de Louis-Napoléon Bonaparte. Ne demeurent que des «victimes politiques", identité parfois revendicative et revancharde, mais qui à terme rompt avec le passé révolutionnaire quand vient l'heure de l'indemnisation dans les années 1880.

16 C'est aussi que la prise d'armes citoyenne n'entretint pas d'affinité avec le militantisme politique. Les clubistes, les candidats à la représentation nationale étaient avant tout des hommes d'une sociabilité particulière, reproduite en partie dans la proscription après le 2 décembre. Au cœur de notre propos, il y a l'écart croissant entre hommes 
politiques et prise d'armes citoyenne. Ceux parmi eux qui ont offert leurs services à la République n'ont pas vécu les journées révolutionnaires comme les autres citoyenscombattants, c'est vrai en 1848 comme en 1851. Ils sont restés préoccupés de la division du travail politique, de la qualité de leurs relations personnelles, de l'effort d'autocontrôle voire d'autocensure dans les rangs des démocrates-socialistes à partir de 1849. Ils sont demeurés les porte-parole du modèle capacitaire de la citoyenneté et se sont retrouvés en porte à faux vis-à-vis de la prise d'armes dès la révolution de février.

17 La délégation ouvrière qui avait connu de beaux jours en 1848 était restée, quant à elle, préoccupée d'une forme de représentation d'une condition sociale singulière, y compris sous sa forme directement politique via la candidature à l'Assemblée - projet toujours d'actualité durant le Second Empire à la faveur, on le sait, des élections législatives de 1863.

18 C'est l'officier de la garde nationale démocratisée, élu par les hommes de sa compagnie, qui disparut avec l'apprentissage de l'illégitimité politique de la prise d'armes citoyennes. Son influence, il la devait à sa participation physique au maintien de l'ordre sorti de la révolution de février, à sa co-présence dans l'événement auprès de ses hommes, aux risques encourus à égalité avec ceux qu'il commandait de perdre la vie au combat. Représentant de la "société civile» en armes au service de la République, il témoignait d'une forme de délégation plus civique que strictement politique, devant tout aux circonstances et donc obligé de s'effacer une fois celles-ci en contradiction avec sa légitimité.

19 Les citoyens-combattants, ces autres quarante-huitards, à Paris les plus nombreux, ne furent pas à l'origine d'un modèle politique fondateur; ils furent les vaincus d'une République qui les désarma. Leurs expériences restèrent pour l'essentiel intransmissibles.

\section{INDEX}

Mots-clés : 2003

these 2003 